Research Paper

\title{
A real-world study in advanced non-small cell lung cancer with de novo brain metastasis
}

\author{
Lei Lei ${ }^{1 \#, ~ W e n-x i a n ~ W a n g 1 \#, ~ D o n g ~ W a n g 2 \#, ~ L i ~ L i n ~} 3$, You-cai Zhu4 ${ }^{4}$, Hong Wang5, Li-ping Wang6, Wu \\ Zhuang7 ${ }^{7}$, Mei-yu Fang1, Bing Wan ${ }^{8 凶}$, Hui-jing Feng ${ }^{9 凶}$, Chun-wei $\mathrm{Xu}^{2^{\circledR}}$
}

1. Department of Chemotherapy, Institute of Cancer Research and Basic Medical Sciences of Chinese Academy of Sciences, Cancer Hospital of University of Chinese Academy of Sciences, Zhejiang Cancer Hospital, Hangzhou Zhejiang 310022, People's Republic of China

2. Department of Respiratory Medicine, Affiliated Jinling Hospital, Medical School of Nanjing University, Nanjing, Jiangsu 210002, People's Republic of China

3. Department of Oncology, Peking University International Hospital, Beijing 102206, People's Republic of China

4. Department of Thoracic Disease Center, Zhejiang Rongjun Hospital, Jiaxing Zhejiang 314000, People's Republic of China

5. Department of Lung Cancer, The Fifth Medical Center, General Hospital of PLA, Beijing 100071, People's Republic of China

6. Department of Thoracic Oncology, Baotou Cancer Hospital, Baotou Inner Mongolia 014000, People's Republic of China

7. Department of Medical Oncology, Fujian Cancer Hospital, Fujian Medical University Cancer Hospital, Fuzhou Fujian 350014, People's Republic of China

8. Department of Respiratory, The Affiliated Jiangning Hopsital of Nanjing Medical University, Nanjing Jiangsu 210002, People's Republic of China

9. Department of Thoracic Oncology, Shanxi Academy of Medical Sciences, Shanxi Bethune Hospital, Taiyuan Shanxi 030032, People's Republic of China

\#Equal contributors

$\triangle$ Corresponding authors: Bing Wan, M.D. \& PhD. Department of Respiratory, The Affiliated Jiangning Hospital of Nanjing Medical University, Nanjing, Jiangsu 211103, 169 Hushan Road, Nanjing, Jiangsu Province, 211103, P.R. China. Phone: +86-25-52087000; Fax: +86-25-52087000; E-mail: bingwan76@163.com. Hui-jing Feng, M.D. \& PhD. Department of Oncology, Shanxi Academy of Medical Sciences, Shanxi Bethune Hospital, Taiyuan Shanxi 030032,99 Longcheng Street, Taiyuan, Shanxi Province, 030032, P.R. China. Phone: +86- 0351-8379598; Fax: +86- 0351-8379131; E-mail: doctorfhj@sina.com. Chun-wei Xu, M.D. \& PhD. Department of Respiratory Medicine, Jinling Hospital, Nanjing University School of Medicine, Nanjing, Jiangsu 210002, 305 Zhongshan Road, Nanjing, Jiangsu Province, 210002, P.R. China. Phone: +86-25-8086-3591; Fax: +86-25-8086-3591; E-mail: dg1935057@smail.nju.edu.cn

(c) The author(s). This is an open access article distributed under the terms of the Creative Commons Attribution License (https://creativecommons.org/licenses/by/4.0/). See http://ivyspring.com/terms for full terms and conditions.

Received: 2020.08.02; Accepted: 2020.12.15; Published: 2021.01.01

\begin{abstract}
Brain metastases are the major cause of life-expectancy shortened for patients with lung cancer. The prognostic value of EGFR mutation subtypes and survival benefit of EGFR-tyrosine kinase inhibitors (TKIs) in advanced non-small cell lung cancer (NSCLC) patients with de novo brain metastasis is still not clear. Here, we present a real-world study nation-wide focusing on the prognostic value of genomic and therapeutic factors in overall survival (OS) of those patients. We enrolled a total of 233 patients diagnosed with advanced NSCLC and de novo BM from multi-medical centers across China. The enrolled patients were divided into 4 groups, including EGFR 19del, EGFR L858R, EGFR wild-type, and EGFR unknown groups. The median OS of patients with EGFR mutations and all patients were 29.0 and 25.0 months, respectively. There was significant difference in OS of patients among EGFR 19del $(n=76)$, EGFR L858R $(n=94), E G F R$ wild-type $(n=46)$ and EGFR unknown $(n=17)$ groups ( 30.5 vs 27.5 vs 16.0 vs $25.0, P=0.025$ ). Patients treated by icotinib showed better $O S$ than gefitinib and erlotinib (31.0 vs 25.5 vs $26.5, \mathrm{P}=0.02)$. There was a difference in OS of patients received the whole-brain radiotherapy (WBRT), stereotactic radiosurgery (SRS), or WBRT+SRS (20.0 vs 31.0 vs 30.0 months, $P<0.001)$, respectively. In multivariate analysis, patients treated with icotinib had superior iPFS benefit than gefitinib and erlotinib $(\mathrm{HR}=0.86[95 \% \mathrm{Cl}(0.74-1.0)], \mathrm{P}=0.04)$. Besides, the histology of non-adenocarcinomas, the number of $\mathrm{BM}(>3)$, and extracranial metastases status could have an independent negative impact on the OS of all patients $(\mathrm{P}<0.001)$. EGFR mutant NSCLC patients with de novo BM had a better OS than patients with EGFR wild type. Patients treated with icotinib had longer iPFS than gefitinib and erlotinib but not in OS. Non-adenocarcinomas, number of BM $(>3)$ and extracranial metastases were independent negative prognostic factors in iPFS and OS of all patients. Prospective clinical trials are warranted to explore more effective multimodality in this population.
\end{abstract}

Key words: de novo brain metastasis, EGFR, EGFR-tyrosine kinase inhibitors (TKIs), non-small-cell lung cancer

\section{Introduction}

Brain metastases are the major cause of the poor prognosis and quality of life in all metastatic cancer patients [1, 2], especially in advanced non-small cell lung cancer (NSCLC) [3]. About $10-35 \%$ of the NSCLC 
patients have de novo $\mathrm{BM}$ on the diagnosis of lung cancer, and more than $50 \%$ of patients would develop BM during the treatment course [4, 5]. The conventional treatment options for advanced NSCLC patients with de novo BM include surgical resection, radiotherapy, and chemotherapy with a median overall survival (OS) that ranges from 4.0-31.0 months [6-8]. Although the combination or sequential use of those therapies have been reported to improve the OS, the major hindrance for chemotherapy is still the barely penetrated blood-brain barrier (BBB) in terms of the big molecular weights of those drugs $[9,10]$.

The identification of oncogene-addicted NSCLC and the development of targeted therapy, like EGFR mutations and EGFR-TKIs, have launched the new era in advanced NSCLC [11]. EGFR-TKIs have shown have remarkable intracranial activity and acceptable tolerance, and survival benefit in advanced NSCLC patients with $\mathrm{BM}$ [12]. It has been shown that $\mathrm{BM}$ occurrence is more frequent in NSCLC patients with EGFR mutations $[13,14]$. Besides, the BM pattern and clinical outcome of NSCLC patients with BM could have an association with EGFR mutation subtypes [15-17]. Of note, the efficacy of TKIs could also be heterogeneous in terms of evidence of low penetration rates through BBB among first- and second generations of TKIs [18, 19], but the increasing potential of penetration by combined therapy, such as radiotherapy [20].

However, most of the research has been conducted in NSCLC patients with metastatic BM in general or limited numbers of patients with de novo $\mathrm{BM}$. The prognostic factors and survival benefit from updated therapies are worth to be discussed in this particular population who are treatment-naïve and longer survival could be expected $[8,21]$. In that case, we designed this real-world study to investigate the prognostic value of EGFR mutation subtypes and the efficacy of TKIs in NSCLC patients with de novo BM from multi-medical centers across China.

\section{Materials and methods}

\section{Patient population}

This study enrolled 233 patients diagnosed as NSCLC with de novo BM from multi-center hospitals in the southeast of China between October 2012 and October 2016. Inclusion criteria were: 1) all the diagnoses were pathologically confirmed on the primary or metastatic tumor using transthoracic needle biopsy or bronchoscopic biopsy, 2) EGFR mutations were detected from the primary or metastatic lesion of all patients, and 3) complete data of baseline clinicopathologic characteristics including age at diagnosis, gender, histology, number of intracranial metastasis, extracranial metastatic status, and ECOG performance status (PS). Exclusion criteria were: 1) patients with other malignant disease histories at the time of diagnosis (due to difficult calculations of recurrent events and double cancers may increase the risk of recurrence), 2) patients who received palliative surgery of primary tumor or metastatic BM were excluded (due to the small sample size and potential longer survival may confound the conclusion of this study).

All patients were grouped by the results of EGFR mutation testing, and patients with EGFR mutations took gefitinib, erlotinib, or icotinib as the first-line targeted therapy if they are asymptomatic BM at the initial diagnosis. Patients with symptomatic BM received radiotherapy as the first-line therapy and followed by systemic therapy (targeted or chemotherapy) according to the genomic and diagnostic characters. The regimens of chemotherapy were classified into pemetrexed-based and non-pemetrexed-based groups. Disease progression was determined based on the radiographic evidence according to Response Evaluation Criteria in Solid Tumors (RECIST) version 1.1. This retrospective study was approved by the ethical committee. All patients' follow-up information was obtained from their last clinical visits, follow-up registration records, and follow-up phone records. The study was approved by Shanxi Academy of Medical Sciences, Shanxi Bethune Hospital Ethics Committee (NO.: YXLL-2015-004) and written informed consent was taken from all the patients.

\section{Study design}

We have collected the clinicopathologic characteristics and survival information from all patients and compared patients in different groups of EGFR mutation subtypes, including EGFR 19del, EGFR L858R, EGFR wild-type, and EGFR unknown groups. The clinicopathologic characteristics of patients were categorized by age $(<65, \geq 65$ years), gender (male, female), ECOG PS (0-1, 2-3), smoking status (never-smoker, smoker), primary tumor histology (non-adenocarcinoma, adenocarcinoma), number of BMs (limited, diffuse), extracranial metastases (yes or no), radiotherapy (WBRT, SRS, or combination of WBRT+SRS), EGFR-TKIs (gefitinib, erlotinib, icotinib), chemotherapy (non-pemetrexedbased, pemetrexed-based).

For the survival analysis, we mainly focus on comparing the correlation with EGFR mutation subtypes and EGFR-TKIs with the iPFS and OS of all patients, as well as other prognostic factors. The iPFS was defined as the duration of time from initial first-line treatment to intracranial progression. The OS 
was defined as the duration of time from the initial diagnosis until death or the most recent follow-up.

\section{Statistical Analysis}

Chi-square test was used to test the association between the EGFR mutation subgroups and clinical categorical variables in all patients. Kaplan-Meier survival analysis and the log-rank test were used to compare the differences in iPFS and OS among patients with different characteristics. These survival comparisons were stratified by EGFR mutation status separately. Multivariate Cox regression was used to determine the independent prognostic factors for iPFS and OS of all patients adjusted by EGFR mutation subtypes, smoking status, primary tumor histology, number of BMs, extracranial metastases, radiotherapy and EGFR-TKIs. All statistical analyses were performed using R v.3.4.1.

\section{Results}

\section{Baseline characteristics of patients}

A total of 233 advanced NSCLC patients who were diagnosed with de novo BMs enrolled in the study. The median age of these patients was 56 years (23-84 years), more female than male patients (173 vs 60), more never-smokers than smokers (161 vs 72) and 212 patients $(91 \%)$ had EGFR PS of $0-1$. These cases included 212 (91\%) adenocarcinoma and 21(9\%) non-adenocarcinoma cases. The EGFR 19 del mutation occurred in $32.6(76 \%)$ patients, $40.4(94 \%)$ patients had the EGFR exon 21 L858R mutation, 46 (19.7\%) EGFR wild-type and 17 (7.3\%) EGFR unknown status. A total of 105 (45.1\%) patients received icotinib (125 $\mathrm{mg} / 3$ times daily), 46 (19.7\%) patients gefitinib (250 $\mathrm{mg} / \mathrm{d})$ and $36(15.5 \%)$ erlotinib (150 mg/d). More than half of patients had extracranial metastasis $(134 / 233,57.5 \%)$. Those patients who received upfront WBRT (30Gy/10f/2W), SRS or WBRT+SRS were $113(52.1 \%), 30(13.8 \%)$ and $5(2.3 \%)$, respectively. A total of 70 patients $(30.5 \%)$ were treated with pemetrexed-based chemotherapy (pemetrexed $500 \mathrm{mg} / \mathrm{m}^{2}$ on day 1 , every $3 \mathrm{ws}$ ) as the first-line treatment, while $162 \quad(69.5 \%)$ received non-pemetrexed-based chemotherapy instead. The patient characteristics at baseline are detailed in Table 1.

\section{Survival outcomes}

Survival analysis showed that the median iPFS and OS from diagnosis of BM were 12.0 (3.0-30.0) and 25.0 (6.0-60.0) months for the whole cohort. Patients with EGFR mutations had a significantly longer OS than those patients with EGFR wild-type (29.0 vs 16.0 months, $\mathrm{P}=0.004$ ) in Figure 1A. The significant difference in OS was observed among groups of patients with EGFR 19 del, EGFR exon 21 L858R, and EGFR unknown mutations ( 30.5 vs 27.5 vs 16.0 vs 25.0 months, $\mathrm{P}=0.025)$ in Figure 1B. Patients treated by icotinib showed better OS than gefitinib and erlotinib ( 31.0 vs 25.5 vs $26.5, \mathrm{P}=0.02$ ) in Figure $\mathbf{2 A}$. There was also a difference in OS of patients who received the whole-brain radiotherapy (WBRT), stereotactic radiosurgery (SRS), or WBRT+SRS (20.0 vs 31.0 vs 30.0 months, $\mathrm{P}<0.001)$, respectively in Figure $\mathbf{2 B}$. No difference was found in the OS of patients treated by pemetrexed-based and non-pemetrexed-based chemotherapies (26.0 vs 24.0 months, $\mathrm{P}=0.31$ ) in Figure 2C.

Table 1. Characteristics of NSCLC patients with de novo brain metastasis in baseline $(n=233)$.

\begin{tabular}{|c|c|c|c|c|c|c|}
\hline Characteristic & $\begin{array}{l}\text { Whole } \\
\text { group, } \\
\mathrm{n}=233 \\
(100 \%)\end{array}$ & $\begin{array}{l}\text { EGFR } \\
\text { 19del } \\
\text { group, } \\
n=76 \\
(32.6 \%)\end{array}$ & $\begin{array}{l}\text { EGFR } \\
\text { 21L858R } \\
\text { group, } \\
\mathrm{n}=94 \\
(40.4 \%)\end{array}$ & $\begin{array}{l}\text { EGFR } \\
\text { wild-type } \\
\text { group, } \\
\mathrm{n}=46 \\
(19.7 \%)\end{array}$ & $\begin{array}{l}\text { EGFR } \\
\text { unknown } \\
\text { group, } \\
\mathrm{n}=17 \\
(7.3 \%)\end{array}$ & $\begin{array}{l}\mathrm{P} \\
\text { value }\end{array}$ \\
\hline Age & & & & & & 0.413 \\
\hline$<65$ & 189(81.1) & $63(82.9)$ & $73(77.7)$ & $37(80.4)$ & 16(94.1) & \\
\hline$\geq 65$ & $44(18.9)$ & 13(17.1) & $21(22.3)$ & $9(19.6)$ & $1(5.9)$ & \\
\hline Gender & & & & & & 0.971 \\
\hline male & $60(25.8)$ & $19(25)$ & $25(26.6)$ & $11(23.9)$ & $5(29.4)$ & \\
\hline female & $173(74.2)$ & $57(75)$ & $69(73.4)$ & $35(76.1)$ & 12(70.6) & \\
\hline ECOG PS & & & & & & 0.165 \\
\hline $0-1$ & 212(91) & $68(89.5)$ & $85(90.4)$ & $44(95.7)$ & 15(88.2) & \\
\hline $2-3$ & $21(9)$ & $8(10.5)$ & $9(9.6)$ & $2(4.3)$ & $2(11.8)$ & \\
\hline Smoking & & & & & & $<0.001$ \\
\hline No & 161(69.1) & $60(78.9)$ & $64(68.1)$ & $21(45.7)$ & 16(94.1) & \\
\hline Yes & $72(30.9)$ & $16(21.1)$ & $30(31.9)$ & $25(54.3)$ & $1(5.9)$ & \\
\hline Histology & & & & & & $<0.001$ \\
\hline${ }^{*}$ Non-adenocarcinoma & $21(9)$ & $0(0)$ & $3(3.2)$ & 18(39.1) & $0(0)$ & \\
\hline Adenocarcinoma & 212(91) & $76(100)$ & $91(96.8)$ & $28(60.9)$ & $17(100)$ & \\
\hline \#Number of BM & & & & & & 0.644 \\
\hline Limited & $120(51.5)$ & $40(52.6)$ & $44(46.8)$ & $26(56.5)$ & $10(58.8)$ & \\
\hline Diffuse & $113(48.5)$ & $36(47.4)$ & $50(53.2)$ & $20(43.5)$ & $7(41.2)$ & \\
\hline Extracranial metastasis & & & & & & 0.531 \\
\hline No & $99(42.5)$ & $33(43.4)$ & $37(39.4)$ & 19(41.3) & $10(58.8)$ & \\
\hline Yes & $134(57.5)$ & $43(56.6)$ & $57(60.6)$ & $27(58.7)$ & $7(41.2)$ & \\
\hline \&Radiotherapy & & & & & & 0.007 \\
\hline No & $69(31.8)$ & $27(38)$ & $30(33.7)$ & $11(26.2)$ & $1(6.6)$ & \\
\hline WBRT & $113(52.1)$ & $36(50.7)$ & $50(56.2)$ & $20(47.6)$ & $7(46.7)$ & \\
\hline SRS & $30(13.8)$ & $6(8.5)$ & $7(7.9)$ & $10(23.8)$ & $7(46.7)$ & \\
\hline WBRT+SRS & $5(2.3)$ & $2(2.8)$ & $2(2.2)$ & $1(2.4)$ & $0(0)$ & \\
\hline EGFR-TKIs & & & & & & $<0.001$ \\
\hline No & $46(19.7)$ & $0(0)$ & $0(0)$ & $46(100)$ & $0(0)$ & \\
\hline Gefitinib & $46(19.7)$ & 14(18.4) & $26(27.7)$ & $0(0)$ & $6(35.3)$ & \\
\hline Erlotinib & $36(15.5)$ & 14(18.4) & $16(17)$ & $0(0)$ & $6(35.3)$ & \\
\hline Icotinib & $105(45.1)$ & $48(63.2)$ & $52(55.3)$ & $0(0)$ & $5(29.4)$ & \\
\hline Chemotherapy & & & & & & 0.453 \\
\hline Non-pemetrexed-based & $162(69.5)$ & $54(71.1)$ & $69(73.4)$ & $29(63)$ & $10(58.8)$ & \\
\hline Pemetrexed-based & $71(30.5)$ & $22(28.9)$ & $25(26.6)$ & $17(37)$ & $7(41.2)$ & \\
\hline \multicolumn{7}{|c|}{$\begin{array}{l}\text { "Non-adenocarcinoma included } 3 \text { cases with adeno-squamous carcinoma, } 11 \\
\text { squamous carcinoma, } 7 \text { non-small cell lung cancer with unknown histologic } \\
\text { subtype. }\end{array}$} \\
\hline $\begin{array}{l}\text { "Number of BM include } \\
\text { ( }>3 \text { intercranial lesions). }\end{array}$ & d the limit & ted BM & -3 inter & anial lesic & s) and dif & se BM \\
\hline
\end{tabular}

In the multivariate analysis, the prognosis of iPFS and OS in all advanced NSCLC patients with de novo BM were not correlated with the EGFR mutation subtype $\quad(\mathrm{HR}=0.92 \quad[95 \% \mathrm{CI} \quad(0.75-1.13)], \quad \mathrm{P}=0.428)$. Whilst those patients who received EGFR-TKIs 
treatment of icotinib had the better outcome of iPFS than gefitinib and erlotinib ( $\mathrm{HR}=0.86$ [95\% CI (0.74-1.0)], $\mathrm{P}=0.04)$ but a trend in OS $(\mathrm{HR}=1.15[95 \% \mathrm{CI}$ (0.99-1.33)], $\quad \mathrm{P}=0.072)$. Also, the histology of non-adenocarcinomas, the number of $\mathrm{BM}(>3)$, and extracranial metastases status had independent negative impacts on the OS of all patients $(\mathrm{P}<0.001)$. All these data were shown in Table 2.
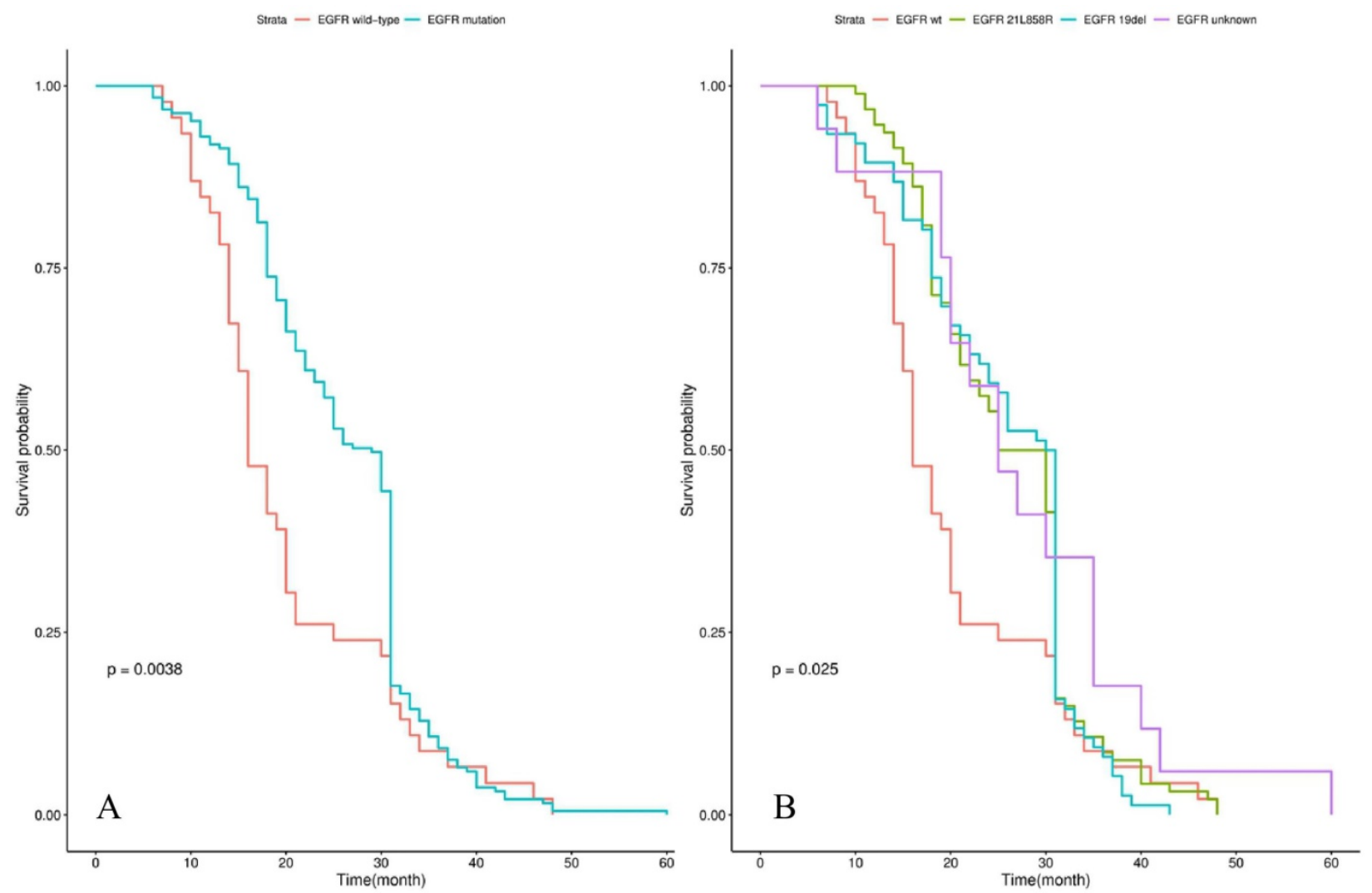

Figure 1. Kaplan-Meier curve analyses of OS in all NSCLC patients with de novo brain metastases. Kaplan-Meier curve was stratified by EGFR mutation status (EGFR wild-type vs EGFR mutation) in A; EGFR mutation types (exon 21 L858R vs exon 19 deletion vs unknown mutation) in B. Abbreviations: OS, overall survival; NSCLC, non-small cell lung cancer; EGFR, epidermal growth factor receptor.
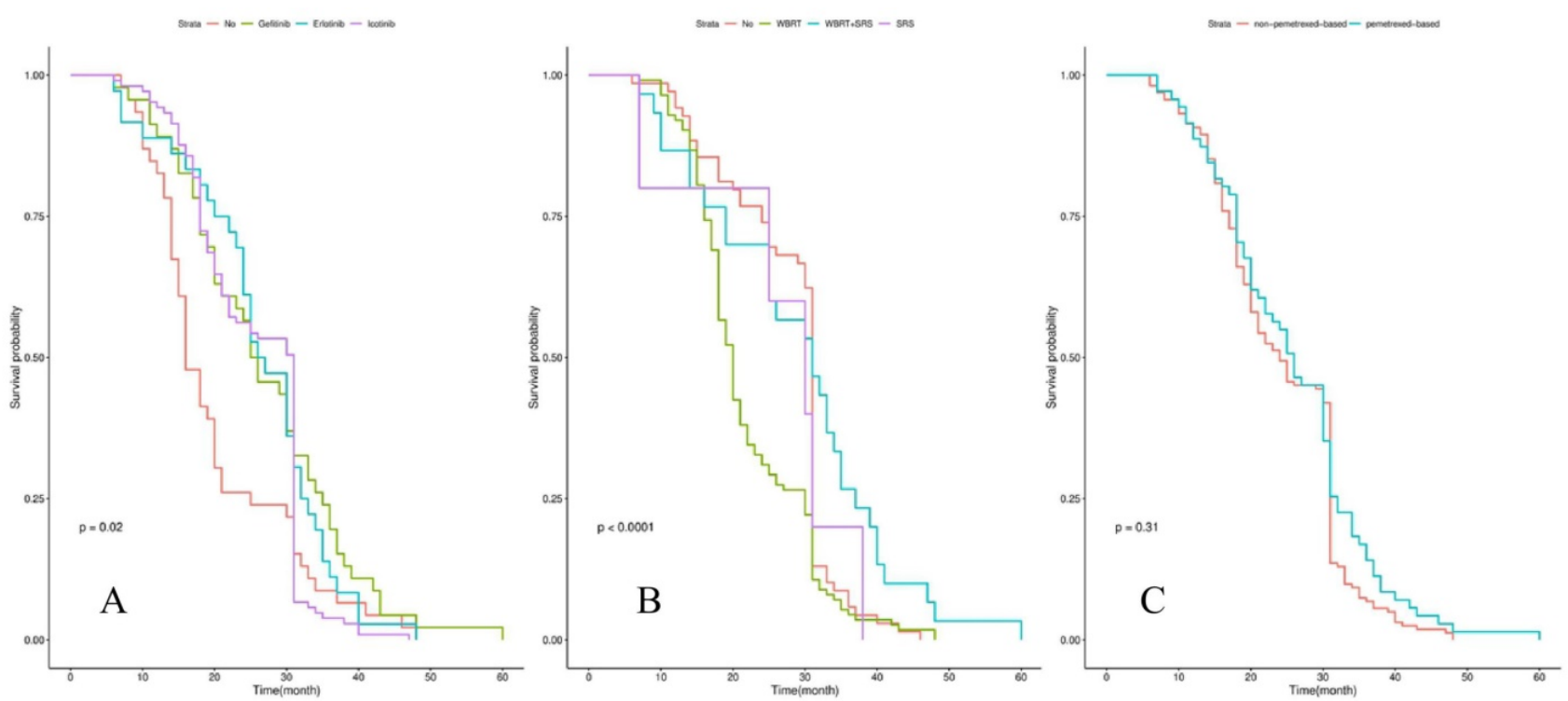

Figure 2. Kaplan-Meier curve analyses of OS in NSCLC patients with de novo brain metastases after EGFR-TKI, radiotherapy and chemotherapy. Kaplan-Meier curve was stratified by EGFR-TKI (gefitinib vs erlotinib vs icotinib) in A; radiotherapy (WBRT vs WBRT+SRS vs SRS) in B; chemotherapy (non-pemetrexed-based vs pemetrexed-based) in C. Abbreviations: OS, overall survival; NSCLC: non-small cell lung cancer; EGFR-TKI, epidermal growth factor receptor-tyrosine kinase inhibitor; WBRT, whole brain radiation therapy; SRS, stereotactic radiosurgery. 
Table 2. Multivariate Cox regression analyses of iPFS and OS

\begin{tabular}{lllllll}
\hline Variable & iPFS & \multicolumn{5}{c}{ OS } \\
\cline { 2 - 7 } & P-value & HR & $95 \%$ CI & P-value & HR & $95 \%$ CI \\
\hline EGFR Mutation & 0.864 & 0.98 & $0.81-1.2$ & 0.428 & 0.92 & $0.75-1.13$ \\
Smoking & 0.422 & 1.14 & $0.83-1.57$ & 0.718 & 1.06 & $0.77-1.46$ \\
Histology & 0.032 & 0.54 & $0.31-0.95$ & $<0.001$ & 0.29 & $0.16-0.51$ \\
$\begin{array}{l}\text { Number of BM } \\
\text { Extracranial }\end{array}$ & $<0.001$ & 1.97 & $1.47-2.66$ & $<0.001$ & 2.22 & $1.65-2.98$ \\
$\begin{array}{l}\text { metastases } \\
\begin{array}{l}\text { Radiation } \\
\text { therapy }\end{array}\end{array}$ & 0.001 & 1.67 & $1.24-2.24$ & $<0.001$ & 2.17 & $1.58-2.98$ \\
TKI & 0.387 & 0.91 & $0.74-1.12$ & 0.956 & 1 & $0.81-1.22$ \\
\hline
\end{tabular}

\section{Discussion}

The prevalence of BM in NSCLC patients is increasing due to reliable imaging techniques, improved survival after novel regimens, the aging population, and advancing [9, 22]. Although the systemic chemotherapy combined with local-control therapy is still the general discipline for advanced NSCLC patients with BM, compelling data has shown the superior efficacy of TKIs than chemotherapy as the first-line therapy in EGFR mutant patients [23-25]. Based on those promising results, EGFR-TKIs have been recently recommended as the first-line therapy for never-smokers with adenocarcinoma of the lung having asymptomatic synchronous BM. However, the treatment of BM patients remains a big challenge for the unmet long-term survival goal. To our best knowledge, this is one of the largest real-world studies on advanced NSCLC patients with de novo $\mathrm{BM}$ across China focusing on the prognostic impact of the EGFR mutation and TKIs; as well as the clinicopathological characteristics and other therapeutic strategies. Firstly, the patients with different subtypes of EGFR mutations did not differ significantly in OS, but the discrepancy presented between EGFR mutant group and EGFR wild-type group. Secondly, the use of icotinib, one of the first-generation of EGFR-TKIs, was found to correlate with better iPFS of patients with EGFR mutation than other two commercial TKIs and a similar trend in OS. Finally, the histology of non-adenocarcinomas, diffuse BM (>3 intracranial lesions) and Extracranial metastases could have a negative independent impact on the OS in advanced NSCLC patients with de novo BM.

A high incidence of BM in NSCLC has been reported in EGFR mutation (40-60\%) [26] or ALK alteration (about 50\%) carriers [27]. Moreover, the incidence of $\mathrm{BM}$ in patients with EGFR mutation is increasing during follow-up after curative surgery [28]. The possible explanations for high-incidence of BM in oncogenic addiction NSCLC patients include the unique tumor characteristics and the low penetration rate of BBB of target therapy. Hsu et al.
[29] reported a higher incidence of BM in EGFR mutation carriers than EGFR wild-type $(39.2 \%$ vs. $28.2 \%, p=0.038)$ and significantly longer median survival (22.4 months vs. 7.9 months, $\mathrm{p}<0.001$ ) in 534 patients with stage IV NSCLC. In our study, we confirmed the longer survival in EGFR mutant patients than EGFR wild-type which is consistent with the previous study. However, the EGFR mutation subtypes did not have an independent prognostic impact on de novo BM patients. Recently, Wang et al. [16] found the prognostic value of EGFR mutation in BMs and patients with the exon 19 deletion may have longer OS compared with exon 21 L858R mutation (not reached vs 26.5 months, $\mathrm{P}=0.0969$ ). In the previous study in NSCLC with the de novo BM population, the extracranial metastatic foci and the response to treatment are important prognostic factors $[7,30]$. The patients enrolled in this study were mainly lung adenocarcinoma, females, non-smokers, and EGFR mutation carriers. It is not surprising that the histology, diffuse BM, and extracranial metastases could have an independent influence on the OS. Besides, more than half of our patients ever received WBRT as local treatment, and WBRT treated patients have the worst prognosis compared with SRS and a combination of WBRT+SRS. Although we are not trying to address the best timing of radiotherapy for de novo BMs, this finding indicates that the optimization of multimodality could weight more than regular local control for patients with BM [31].

Although the emerging promising efficacy of third-generation TKIs has been approved in advanced NSCLC with BM, the first generation of EGFR-TKIs are still commonly used as the first-line therapy for patients in China. We found that patients received icotinib had a longer median iPFS than patients treated by gefitinib and erlotinib. In a phase III trial (BRAIN), icotinib was associated with a significantly longer iPFS than WBRT combined with chemotherapy [32]. Icotinib (BPI-2009H, Conmana) is an oral quinazoline compound that administered mainly in China for the lower expense, and it has a similar chemical structure to gefitinib and erlotinib [33]. We assume that the superior of icotinib than other TKIs should be verified in further research since they have a similar mechanism of activity and therapeutic effects. The major challenge of TKIs in EGFR mutation patients with BM encountered is acquired drug resistance, which occurs very common in the first and second generation of TKIs [34]. The new generation of TKIs including AZD3759 has been designed to overcome this problem [35]. Regarding the efficacy in treatment and prophylaxis of BM by pemetrexed chemotherapy [36], we also compared the survival benefit of pemetrexed based or non-pemetrexed based 
chemotherapy in all patients including those with EGFR wild-type. No significant difference has been found in the survival analysis but further study is encouraged. Recently, PD-L1 positive NSCLC patients with BM could also benefit from immunotherapy [37]. In summary, further research should continue finding the best therapy after the resistance of first-line TKIs in EGFR mutant BM patients and explore novel therapy for EGFR wild-type BM patients.

There are some limitations should be mentioned in our study. Firstly, we collected all the medical information retrospectively and the potential bias in patient selection could not be avoided. The involvement of surgical resection of primary and metastatic lesions has not been discussed in this study; Secondly, the baseline clinical characteristics of EGFR mutation patients in groups were not well balanced which could lead to the bias of conclusion. Third, the frequency of another important driver gene alteration $A L K$ fusion should be presented due to its correlation with the development of BM in NSCLC patients [27]. However, there were limited $A L K$ detection data in our study and no ALK inhibitor has been administered either due to the lack of insurance coverage and approach for targeted therapy during that period. Finally, those treatments after first-line TKIs could likely influence the long-term survival of patients, as well as the documented adverse events and quality of life were lacking. Therefore, we were unable to analyze these potential prognostic factors in this study.

In summary, we discussed the prognostic influence of clinical characteristics and multimodality on advanced NSCLC patients with de novo BMs in a real-world study. Patients with different EGFR mutation subtypes may have a different response to TKIs which will turn out to have an impact on long-term survival. As the emerging results of novel target therapy and immunotherapy have shed light on the NSCLC patients with BM, larger prospective randomized clinical trials are urgently needed to explore the optimal multimodality in care for de novo $\mathrm{BM}$ patients as well for longer survival.

\section{Acknowledgments}

This study was supported in part by grants from Zhejiang Public Welfare Technology Research Program (LGJ20H160001), Science and Technology Planning project of Zhejiang Province (LGF19H160002), the Medical Scientific Research Foundation of Zhejiang Province of China (2019RC027), Zhejiang Traditional Chinese Medicine Science Fund Project (2020ZB037, 2021ZQ013), Scientific Research Foundation of Zhejiang Medical
Association (2019ZYC-A76), Huilan Public-Hanson Pharmaceutical Lung Cancer Precision Medical Research Special Fund Project Foundation (HL-HS2020-5) and Xisike-Hanson Cancer Research Foundation (Y-HS2019-20).

\section{Author Contributions}

(I) Conception and design: Chun-wei $\mathrm{Xu}$, Wen-xian Wang, Bing Wan, Hui-jing Feng.

(II) Administrative support: Mei-yu Fang, Lei Lei, Dong Wang.

(III) Provision of study materials or patients: Dong Wang, Li Lin, You-cai Zhu, Hong Wang, Li-ping Wang, Wu Zhuang.

(IV) Collection and assembly of data: Chun-wei $\mathrm{Xu}$, Wen-xian Wang, Dong Wang.

(V) Data analysis and interpretation: Lei Lei.

(VI) Manuscript writing: All authors.

(VII)Final approval of manuscript: All authors.

\section{Ethical Statement}

The authors are accountable for all aspects of the work in ensuring that questions related to the accuracy or integrity of any part of the work are appropriately investigated and resolved. Written informed consent was obtained from the patient for publication of this manuscript and any accompanying images. The study was approved by Shanxi Academy of Medical Sciences, Shanxi Bethune Hospital Ethics Committee (NO.: YXLL-2015-004) and written informed consent was taken from all the patients.

\section{Competing Interests}

The authors have declared that no competing interest exists.

\section{References}

1. Bray F, Ferlay J, Soerjomataram I, et al. Global cancer statistics 2018: GLOBOCAN estimates of incidence and mortality worldwide for 36 cancers in 185 countries. CA Cancer J Clin. 2018, 68(6):394-424.

2. Liu $Q$, Tong $X$, Wang J, et al. Management of brain metastases: history and the present. Chin Neurosurg J. 2019, 5:1.

3. Peters S, Bexelius C, Munk V, et al. The impact of brain metastasis on quality of life, resource utilization and survival in patients with non-small-cell lung cancer. Cancer Treat Rev. 2016, 45:139-62.

4. Burt M, Wronski M, Arbit E, et al. Resection of Brain Metastases From Non-Small-Cell Lung Carcinoma. Results of Therapy. Memorial Sloan-Kettering Cancer Center Thoracic Surgical Staff. J Thorac Cardiovasc Surg. 1992, 103(3):399-410.

5. Billing P S, Miller D L, Allen M S, et al. Surgical Treatment of Primary Lung Cancer With Synchronous Brain Metastases. J Thorac Cardiovasc Surg. 2001, 122(3):548-53.

6. Penel N, Brichet A, Prevost B, et al. Pronostic factors of synchronous brain metastases from lung cancer. Lung Cancer. 2001, 33(2-3):143-54.

7. Kong D S, Lee J I, Nam D H, et al. Prognosis of non-small cell lung cancer with synchronous brain metastases treated with gamma knife radiosurgery. J Korean Med Sci. 2006, 21(3):527-532.

8. Lee $\mathrm{V} \mathrm{H}$, Tin V P, Choy $\mathrm{T} \mathrm{S}$, et al. Long-term survival in patients with non-small cell lung cancer and synchronous brain metastasis treated with whole-brain radiotherapy and thoracic chemoradiation. Radiat Oncol. 2011, 6:166

9. Zimmermann S, Dziadziuszko R, Peters S. Indications and limitations of chemotherapy and targeted agents in non-small cell lung cancer brain metastases. Cancer Treat Rev. 2014. 40:716-722. 
10. Fidler I J, Yano S, Zhang R D, et al. The seed and soil hypothesis: vascularisation and brain metastases. Lancet Oncol. 2002, 3(1):53-57.

11. Linardou H, Dahabreh I J, Bafaloukos D, et al. Somatic EGFR mutations and efficacy of tyrosine kinase inhibitors in NSCLC. Nat Rev Clin Oncol. 2009, 6(6):352-366

12. Chiu $\mathrm{C} \mathrm{H}$, Tsai $\mathrm{C} \mathrm{M}$, Chen $\mathrm{Y} M$, et al. Gefitinib is active in patients with brain metastases from non-small cell lung cancer and response is related to skin toxicity. Lung Cancer. 2005, 47:129-138.

13. Eichler A F, Kahle K T, Wang D L, et al. EGFR mutation status and survival after diagnosis of brain metastasis in nonsmall cell lung cancer. Neuro Oncol. 2010, 12(11):1193-9.

14. Li Z, Lu J, Zhao Y, et al. The retrospective analysis of the frequency of EGFR mutations and the efficacy of gefitinib in NSCLC patients with brain metastasis. J Clin Oncol. 2011, 29(Suppl):e18065.

15. Sekine A, Kato T, Hagiwara E, et al. Metastatic brain tumors from non-small cell lung cancer with EGFR mutations: distinguishing influence of exon 19 deletion on radiographic features. Lung Cancer. 2012, 77:64-69.

16. Wang $\mathrm{H}, \mathrm{Yu} X$, Fan $\mathrm{Y}$, et al. Multiple treatment modalities for brain metastasis in patients with EGFR-mutant non-small-cell lung cancer. Onco Targets Ther. 2018, 11:2149-2155.

17. Porta R, Sánchez-Torres J M, Paz-Ares L, et al. Brain metastases from lung cancer responding to erlotinib: the importance of EGFR mutation. Eur Respir J. 2011, 37(3):624-31.

18. Hoffknecht $\mathrm{P}$, Tufman $\mathrm{A}$, Wehler $\mathrm{T}$, et al. Efficacy of the Irreversible ErbB Family Blocker Afatinib in Epidermal Growth Factor Receptor (EGFR) Tyrosine Kinase Inhibitor (TKI)-pretreated Non-Small-Cell Lung Cancer Patients With Brain Metastases or Leptomeningeal Disease. J Thorac Oncol. 2015, 10(1):156-63.

19. Zhao J, Chen M, Zhong W, et al. Cerebrospinal Fluid Concentrations of Gefitinib in Patients With Lung Adenocarcinoma. Clin Lung Cancer. 2013, 14(2):188-93.

20. Fang L, Sun $\mathrm{X}$, Song $\mathrm{Y}$, et al. Whole-brain radiation fails to boost intracerebral gefitinib concentration in patients with brain metastatic non-small cell lung cancer: a self-controlled, pilot study. Cancer Chemother Pharmacol. 2015, 76(4):873-877

21. Flannery $T \mathrm{~W}$, Suntharalingam $M$, Regine $W$ F, et al. Long-Term Survival in Patients With Synchronous, Solitary Brain Metastasis From Non-Small-Cell Lung Cancer Treated With Radiosurgery. Int J Radiat Oncol Biol Phys. 2008, 72(1):19-23.

22. Nayak L, Lee E Q, Wen P Y. Epidemiology of Brain Metastases. Curr Oncol Rep. 2012, 14(1):48-54.

23. Rosell R, Carcereny E, Gervais R, et al. Erlotinib versus standard chemotherapy as first-line treatment for European patients with advanced EGFR mutation-positive non-small-cell lung cancer (EURTAC): a multicentre, open-label, randomised phase 3 trial. Lancet Oncol. 2012, 13(3):239-46.

24. Mok T S, Wu Y L, Thongprasert S, et al. Gefitinib or carboplatin-paclitaxel in pulmonary adenocarcinoma. N Engl J Med. 2009, 361(10):947-57.

25. Yang J C H, Hirsh V, Schuler M, et al. Symptom Control and Quality of Life in LUX-Lung 3: A Phase III Study of Afatinib or Cisplatin/Pemetrexed in Patients With Advanced Lung Adenocarcinoma With EGFR Mutations. J Clin Oncol. 2013, 31(27):3342-50.

26. Han $\mathrm{G}, \mathrm{Bi} \mathrm{J}$, Tan $\mathrm{W}$, et al, A retrospective analysis in patients with EGFR-mutant lung adenocarcinoma: is EGFR mutation associated with a higher incidence of brain metastasis? Oncotarget. 2016, 7(35):56998-57010.

27. Camidge D R, Kim D W, Tiseo M, et al. Exploratory analysis of brigatinib activity in patients with anaplastic lymphoma kinase-positive non-small-cell lung cancer and brain metastases in two clinical trials. J Clin Oncol. 2018, 36(26):2693-2701.

28. Shin D Y, Na I I, Kim C H, et al. EGFR Mutation and Brain Metastasis in Pulmonary Adenocarcinomas. J Thorac Oncol. 2014, 9(2):195-9.

29. Hsu F, Nichol A, Toriumi T, et al. Miliary metastases are associated with epidermal growth factor receptor mutations in non-small cell lung cancer: a population-based study. Acta Oncol. 2017, 56(9):1175-1180.

30. Owen S, Souhami L. The management of brain metastases in non-small cell lung cancer. Front Oncol. 2014, 4:248

31. Magnuson W J, Lester-Coll N H, Wu A J, et al. Management of brain metastases in tyrosine kinase inhibitor-naïve epidermal growth factor receptor-mutant non-small-cell lung cancer: a retrospective multi-institutional analysis. J Clin Oncol. 2017, 35(10):1070-1077.

32. Yang J J, Zhou C, Huang Y, et al. Icotinib versus whole-brain irradiation in patients with EGFR-mutant non-small-cell lung cancer and multiple brain metastases (BRAIN): a multicentre, phase 3, open-label, parallel, randomised controlled trial. Lancet Respir Med. 2017, 5(9):707-716.

33. Guan Y S, He Q, Li M, et al. Icotinib: activity and clinical application in Chinese patients with lung cancer. Expert Opin Pharmacother. 2014, 15(5):717-28.

34. Kelly W J, Shah N J, Subramaniam D S. Management of brain metastases in epidermal growth factor receptor mutant non-small-cell lung cancer. Front Oncol. 2018, 8:208

35. Yang Z, Guo Q, Wang Y, et al. AZD3759, a BBB-penetrating EGFR inhibitor for the treatment of EGFR mutant NSCLC with CNS metastases. Sci Transl Med. 2016, 8:368ra172-368ra172.

36. Ortuzar W, Hanna N, Pennella E, et al. Brain metastases as the primary site of relapse in two randomized phase III pemetrexed trials in advanced non-small-cell lung cancer. Clin Lung Cancer. 2012, 13:24-30.
37. Goldberg S B, Schalper $\mathrm{K}$ A, Gettinger SN, et al Pembrolizumab for management of patients with NSCLC and brain metastases: long-term results and biomarker analysis from a non-randomised, open-label, phase 2 trial. Lancet Oncol. 2020, 21(5):655-663. 